\title{
Desarrollo de estrategias metodológicas que permitan mitigar el miedo escénico en los estudiantes de la IED Víctor Camargo Álvarez ${ }^{1}$ \\ Development of methodological strategies to mitigate stage fright among IED students Victor Camargo Álvarez.
}

DOI: http://dx.doi.org/10.17981/cultedusoc.9.3.2018.09

Artículo de investigación. Fecha de recepción: 15/06/2018. Fecha de aceptación: 27/11/2018

Martha Cabarcas ${ }^{2}$

Emilse Puertas, Orlando Hernández, Mauricio Mizger y Erbinson Caballero ${ }^{3}$

Institución Educativa Departamental Víctor Camargo Álvarez (Colombia)

cabarcasmc@hotmail.com

Para citar este artículo:

Cabarcas, M., Puertas, E., Hernández, O., Mizger, M. y Caballero, E. (2018). Desarrollo de estrategias metodológicas que permitan mitigar el miedo escénico en los estudiantes de la IED Víctor Camargo Álvarez. Cultura. Educación y Sociedad 9(3), 79-84. DOI: http:/l dx.doi.org/10.17981/cultedusoc.9.3.2018.09

\section{Resumen}

Un padecimiento de las relaciones personales, es el miedo escénico o pánico escénico, el cual es un estado inhibitorio que reduce la efectividad comunicacional e impide el despliegue de las capacidades expresivas potenciales de los educandos. Esta investigación se realizó con la intención de mitigar el pánico escénico en los educandos participantes, utilizando distintas estrategias como encuentros literarios y talleres para propiciar el desarrollo de las competencias argumentativas y propositiva en los beneficiarios. Metodológicamente se orientó bajo un enfoque cualitativo, desde un diseño descriptivo y se utilizaron como instrumentos formatos de entrevistas y encuesta; la muestra estuvo conformada por 50 estudiantes de la Institución Educativa Víctor Camargo Álvarez. Los resultados demuestran que algunos estudiantes padecían miedos escénicos por falsas creencias, que ya están superando, aunque queda mucho por trabajar para ayudarlos a mitigar esta falencia que afecta la expresión en público de los estudiantes.

Palabras clave: Miedo escénico, desarrollo de competencias, estudiantes.

\section{Abstract}

A condition of personal relationships, is the stage fright or stage fright, which is an inhibitory state that reduces the communicational effectiveness and prevents the deployment of the potential expressive capacities of the learners. This research was carried out with the intention of mitigating the stage panic in the participating students, using different strategies such as literary meetings and workshops to promote the development of argumentative and proactive competences in the beneficiaries. Methodologically, it was guided by a qualitative approach, from a descriptive design and interviews and survey formats were used as instruments; the sample consisted of 50 students from the Víctor Camargo Álvarez Educational Institution. The results show that some students suffered from stage fears due to false beliefs, which they are already overcoming, although there is still a lot of work to be done to help them mitigate this flaw that affects the public expression of students

Keywords: Stage fright, competence development, students.

1 Este artículo ha sido derivado del Programa de Fortalecimiento de la Cultura Ciudadana y Democrática CT+I a través de la IEP apoyada en TIC en el Departamento de Magdalena: CICLON

2 Líder del grupo de investigación "INEVICAL".

3 Docentes de la Institución Educativa Departamental Víctor Camargo Álvarez y del grupo de investigación "INEVICAL".

- The author; licensee Universidad de la Costa - CUC

Cultura, Educación y Sociedad vol. 9 no. 3, pp. 79-84. Diciembre, 2018

Barranquilla. ISSN 2389-7724 Online 


\section{Introducción}

Es recurrente encontrar en las instituciones educativas, un alto índice de miedo escénico en los estudiantes, en mucho de los casos esto le impide participar en actividades públicas y mostrar sus capacidades. Llegado el momento de exposición pública, las personas o los estudiantes en este caso, pueden presentar dificultades cognitivas como fallos de memoria o incapacidad de razonar correctamente, así como pensamientos negativos sobre la actuación o sobre sí mismos (Alzugaray, Lopez, Hernández, 2016). Lo complejo de esta situación es que, en ocasiones, no reciben ningún tipo de orientación por parte de la institución, de manera que es difícil controlar los síntomas de pánico escénico (Campoverde, 2017).

En los jóvenes, la timidez suele tener repercusión negativa en la evolución de su personalidad, pudiendo generar, inseguridad, traumas o fobias que en algunas ocasiones no les permiten desenvolverse adecuadamente en la sociedad (Terán, 2014). Estos son producidos en gran medida en el hogar o en las instituciones, que son los ambientes donde permanece la mayor parte del tiempo.

Adicional, cuando son estudiantes adultos mayores o con alguna discapacidad aumenta este pánico escénico, es por esto que tomamos la expresión de Araque y Suárez (2017), donde nombran que la ideología cristina influye en mejorar las condiciones de trato a los ciegos, paralíticos, sordos y mudos, ente otros. Es a partir del siglo XVIII cuando la sociedad comienza a entender su responsabilidad hacia los seres humanos más vulnerables tanto física como mentalmente.

\section{El arte escénico}

El arte escénico involucra muchos temas, entre esos encontramos bailes, danzas y música, por lo tanto, desempeña un papel fundamental en la educación de los niños, debido a que en ellos es que se empiezan a desarrollar habilidades. La investigación podría ayudar al proceso de enseñanzaaprendizaje en el aula, y a su vez contribuir específicamente al desarrollo social de los estudiantes, ayudando así a la resolución de problemas que estén relacionados con la capacidad de expresión y adaptación de los niños (Chacón y Garabi, 2017).

La palabra, la voz, el gesto, es decir la comunicación oral es uno delos instrumentos más importantes de que disponen los maestros y las maestras para transferir las enseñanzas a su alumnado. Por otra parte, la comunicación oral del profesorado constituye también el modelo de interacción social y de lenguaje que los niños/as aprenden de manera inductiva. Las razones que llevan a plantear esta idea es que en las escuelas dedican muy poco tiempo al desarrollo de la lengua oral en comparación con la lengua escrita, esto como consecuencia lleva a que los estudiantes sientan temor de equivocarse o decir algo incorrecto al momento de expresarse (Balaguer, Fuentes y Palau, 2015). En el proceso de desarrollo integral de una persona, se requiere de una gran habilidad para la comunicación hablada y escrita, ya que potencia las relaciones interpersonales y promueve el desarrollo de las habilidades cognitivas (Malaver, 2017).

Estrategias como el incentivar a los estudiantes a participar en obras de teatro, podría ayudar de cierta manera que estos pierdan el miedo al escenario, ya que el teatro posee códigos, lenguajes, estilos, recursos, y requiere de habilidades y capacidades especiales, incluso podría decirse "un don" especial, ósea que es un terreno que le pertenece a los actores o sea a los artistas y del cual el resultado final está en la puesta en escena ante un escenario con su correspondiente exigencia. Podemos decir que el teatro ha sido creado para motivar e incentivar a las personas a desarrollar habilidades y mostrarse ante al público con diversas cualidades (Gerena, Absalon, Arias, 2015).

Los maestros para contribuir a este hecho piensan en desarrollar actividades que 
hagan relación entre el hecho escénico y la clase: el actor es el emisor, el público son los estudiantes y el docente; y el espacio escénico es el salón. El emisor establece una lógica interacción con sus estudiantes a partir de su cuerpo, el contenido de su clase y el tipo de relación que quiera establecer con ellos en donde implica comunicación verbal y no verbal (Alvarado, 2016). La aplicación del teatro como estrategia didáctica, en la cual, el maestro se basará para lograr una buena comunicación asertiva con los estudiantes de educación básica, siendo una herramienta interactiva, la cual ayudará a reforzar todos los conocimientos adquiridos, convirtiéndose en aprendizajes significativos.

En estudios encontrados, se encuentra que un alto porcentaje de docentes están dispuestos a mejorar y capacitarse en forma permanente, también un porcentaje de padres comprometidos con la educación de sus hijos, ejecución de proyectos educativos con la finalidad de desarrollar la expresión oral, previsión de situaciones significativas teniendo en cuenta el enfoque comunicativo textual (Díaz, 2017). Esto se evidenciará cuando los estudiantes sobresalgan en determinadas manifestaciones artísticas, pero no en las tradicionales. Todo esto en ocasiones lleva a que los padres de los estudiantes consideren que sus hijos pierden su tiempo y no aprenden asignaturas como matemáticas, lenguaje, etc. las cuales les servirá para la vida futura (Ibarra y $\mathrm{Mu}$ rillo, 2013).

El conocimiento de la importancia y beneficios de la expresión corporal para una excelente interpretación escénica, mucho más allá del conocimiento científico tecnológico que obtienen los estudiantes en las aulas que es importante para su desarrollo práctico profesional, pues esto los ayuda a enriquecer su personalidad como tal, les proporciona herramientas que faciliten el desarrollo de su carácter y entre otras habilidades, los forma también de tal manera que pueden desenvolverse en una sociedad (Palacios, 2014).

\section{Pánico escénico en estudiantes}

El miedo escénico es, con frecuencia, uno de los problemas con los que se enfrenta una persona, estudiante o profesional en el desarrollo de su habilidad, la ansiedad desproporcionada que en algunos sujetos produce una puesta en escena se manifiesta en actuaciones de muy diversa índole (conciertos, conferencias, competiciones deportivas, etc.).Cuando define el miedo escénico como la ansiedad que produce la posibilidad de cometer errores delante de la audiencia en una interpretación y que evoca sentimientos de vergüenza y humillación (Llaneza y Larrinaga, 2017).

Yagosesky, define al Miedo Escénico como: "La respuesta psicofísica del organismo, generalmente intensa, que surge como consecuencia de pensamientos anticipatorios catastróficos sobre la situación real o imaginaria de hablar en público" (González, 2016). Los docentes expresan su preocupación por el desenvolvimiento inadecuado de los estudiantes no solo en las exposiciones en clase sino también en las habilidades interpersonales, al momento de encontrarse frente a un público el miedo los toma y evita que estos puedan expresarse de manera adecuada, por esto es necesario que en clase se desarrollen habilidades que ayuden a los estudiantes a poder superar sus miedos (Rodríguez, 2015).

El dominio que tenga el estudiante frente a un público al momento de querer exponer o explicar una idea, se ha convertido en un punto importante en el desempeño del estudiante, pues esto se encuentra relacionado con la capacidad de expresar sus ideas (Alava,2017). El nerviosismo es un tema clave y muy recurrente en las conversaciones entre los estudiantes, debido a esto, se pretende lograr que el maestro redescubra la confianza interior que existe en el alumno, de manera que así puede enseñarlo a disfrutar en el escenario, a mejorar su relación con el público y su estudio cotidiano (Pignatelli, 2015).

Algunos docentes al momento de enseñar las clases tienen la tendencia de solo dictar, 
de no hacer algo diferente y creativo, del juego escénico a la puesta en escena es una metodología y a la vez una herramienta que de cierta forma contribuirá al desarrollo de destrezas habilidades y perdida del temor por parte del estudiante al presentarse frente a los demás (Alexandre, Cáceres, 2015). Es importante escuchar al estudiante, valora sus palabras y permitir expresar sus justificaciones.

\section{Metodología}

Se realizó un estudio de tipo cualitativo, desde un diseño descriptivo, La investigación cualitativa busca conocer e interpretar la realidad de los participantes a través de sus propias experiencias, entregando una información subjetiva del fenómeno de estudio. (Hernández, Fernández, y Baptista, 2010). Los estudios con un diseño descriptivo están encaminados a especificar las características de la población sujeto de estudio o los fenómenos que sean sometidos a análisis (Hernández, Fernández, y Baptista, 2010).

\section{Población y muestra}

En cuanto a la población beneficiada con el estudio, se tomó una muestra representativa de cincuenta (50) estudiantes de segundo a cuarto grado de la I.E.D Víctor Camargo Álvarez, con el objetivo de disminuir el medo que presentan los estudiantes al momento de presentarse o exponer algún tema frente a un grupo de personas, por medio de los encuentros literarios, al tiempo que los estudiantes van desarrollando competencias discursivas y argumentativas que les ayuden a desenvolverse mejor en sociedad.

\section{Instrumentos}

Para llevar a cabo la investigación, se aplicaron encuestas y se realizaron entrevistas semiestructuradas a los 50 estudiantes participantes, con la intención de conocer las principales causas y creencias que permiten el miedo escénico. También se utilizó un registro con diario de campo para realizar las anotaciones importantes, tanto en la implementación como al finalizar el proceso.

\section{Resultados}

A continuación, se presentan en la tabla 1, los principales hallazgos del estudio organizado por momentos.

TABLA 1

Resultados importantes por momentos.

\begin{tabular}{|c|c|c|}
\hline \multicolumn{2}{|c|}{ Momento } & Hallazgos importantes \\
\hline $\begin{array}{c}\text { Momento } \\
1\end{array}$ & Diagnóstico & $\begin{array}{l}\text { Se realizaron entrevistas y se aplicaron encuestas a } 50 \text { de estudiantes de } \\
\text { la I.E.D Víctor Camargo, para conocer acerca de los miedos, temores de los } \\
\text { estudiantes así como también sus opiniones acerca de los pensamientos y } \\
\text { creencias que presentan cuando se deben expresarse en público. }\end{array}$ \\
\hline $\begin{array}{c}\text { Momento } \\
2\end{array}$ & $\begin{array}{l}\text { Diseño de } \\
\text { estrategias }\end{array}$ & $\begin{array}{l}\text { De acuerdo a la característica de la población, se diseñaron talleres } \\
\text { prácticos, encuentros literarios, clases de oratoria, entre otras estrategias } \\
\text { para mitigar el miedo entre los estudiantes. Una de las estrategias se basó } \\
\text { en derrumbar falsas creencias de la exposición al público. }\end{array}$ \\
\hline $\begin{array}{c}\text { Momento } \\
3\end{array}$ & $\begin{array}{l}\text { Imple- } \\
\text { mentación }\end{array}$ & $\begin{array}{l}\text { Se implementó durante } 3 \text { meses las estrategias diseñadas, en el colegio, } \\
\text { teniendo los estudiantes y profesores un papel activo. Los estudiantes se } \\
\text { divertían mucho durante los talleres prácticos, aprendiendo que muchos } \\
\text { de sus pensamientos estaban errados y que tenían muchas habilidades y } \\
\text { competencias. }\end{array}$ \\
\hline
\end{tabular}


Las mediciones evidenciaron que el $75 \%$ de los estudiantes temen a expresarse en público por burlas de sus compañeros, un 36\% manifestó tener capacidades para expresarse; un $45 \%$ de los educandos entrevistados y encuestados han presentado problemas y/o síntomas relacionados con el miedo escénico, el 35\% de los encuestados y entrevistados manifestó que casi siempre presenta síntomas, frente a un $15 \%$ que manifiesta que lo ha sufrido siempre y un $5 \%$ que nunca ha tenido síntomas ni ha presentado dificultades relacionadas con el miedo escénico.

Luego de la implementación de las estrategias, el porcentaje que mostró la mayor diferencia fue en cuanto al temor de burlas, que bajó a $40 \%$ y las capacidades para expresarse que aumentó a un $65 \%$.

\section{Discusión}

El miedo escénico, es una problemática identificada en los estudiantes de la I.E.D Víctor Camargo de Álvarez, que necesitaba intervención por parte de la comunidad educativa pues por causa de esto, los estudiantes tenían muchos miedos inculcados que no lo permitían expresarse con mayor libertad. La expresión de sus ideas permite desarrollar sus conocimientos y potencial, por esta razón el grupo de investigación INEVICAL realizó la propuesta de encuentros literarios como herramienta de innovación e investigación para mitigar el miedo escénico, con el fin de comenzar a dar respuesta a las problemática. Se realizaron aproximaciones y diferentes actividades a través de los encuentros literarios lo que ha permitido que los estudiantes puedan ir perdiendo el miedo de hablar en público, y evitando pensamientos autosaboteadores cuando se disponen a expresarse frente a un grupo de personas.

No obstante, se hace necesario seguir trabajando y generar prácticas educativas que permitan disminuir el padecimiento de los estudiantes, desde el aula de clase, hasta llevarlos a la participación en actos cívicos escolares. A través de los encuentros literarios se buscó promover, además descubrimiento de capacidades, habilidades, competencias y actitudes de tolerancia, colaboración, cooperación y solidaridad en la comunicación con los demás miembros. Estas actividades lúdicas se involucraron de forma transversal en las diferentes áreas y asignaturas de los planes de estudio de la I.E.D Víctor Camargo Álvarez.

\section{Referencias}

Acevedo, C. (2017). Las tiendas de barrio desde la economía institucional. IJMSOR, 2(1), 30-37. Recuperado de http://ijmsoridi.com/ index.php/ijmsor/article/view/85

Alexandre, M., Luis, J. y Cáceres Bajaña, M. D. P. (2015). Del juego escénico a la puesta en escena como aprendizaje teatral en los estudiantes de 8vo año EGB y docentes de la Unidad Educativa Fiscal Ficoa de Montalvo ubicado en el sector norte de la ciudad de Guayaquil. [Tesis de maestría]. Universidad de Guayaquil, Guayaqui.

Alzugaray, Z., López, Ó. y Hernández, S. (2016). Estudios de música en los conservatorios superiores y ansiedad escénica en España. Revista Electrónica Complutense de Investigación en Educación Musical, 13. 50.

Araque, F. y Suárez, O. (2017). Equidad ÉticaJurídica de la Ciencia, para la Emancipación del Conocimiento y los Saberes. Jurídicas CUC, 13(1). 97-120. DOI: http:// dx.doi. org/10.17981/juridcuc.13.1.2017.5

Balaguer M., Fuentes M. y Palau M. (2015). La competencia comunicativa oral en la formación inicial de maestros y maestras. $O p$ ción, 31(5).

Campoverde, C. (2017). Comunicación visual y su influencia en la elaboración de material impreso con técnicas para controlar el pánico escénico y ayuda a la integración de estudiantes de octavo paralelo " $a$ " $y$ " $b$ " del colegio mixto" Leonidas Garcia”. [Tesis de doctorado]. Universidad de Guayaquil, Guayaqui. 
Chacón, D., Garabi, R. y Pilar, M. (2017). La influencia de las artes escénicas como base en el desarrollo social de los niños de 4 a 5 años, en la escuela Rafaela Vallejo Barahona, período lectivo 2016-2017. [Tesis pregrado]. Universidad de Guayaquil, Guayaqui.

De La Cruz, I. y Colina, C. (2015). El teatro como estrategia didáctica en el fortalecimiento de la comunicación asertiva en los estudiantes del cuarto año de educación básica de la escuela "España" de la parroquia la Concepción del Aantón ambato de la provincia del Tungurahua. [Tesis maestría].

Diaz, D., Nina, M. y Carla, M. (2017). Estrategias lúdicas para desarrollar la expresión oral en los niños y niñas de 5 años de la Institución Educativa Inicial La Pampa, Camaná, 2016.

Hernández, D. y Escobar, A. (2017). Modelo de contabilidad social como herramienta de gestión para la responsabilidad social empresarial. IJMSOR, 2(1), 44-56. Recuperado de http://ijmsoridi.com/index.php/ijmsor/article/ view/86

Ibarra, C. y Murillo, K. (2013). Materiales del entorno para el desarrollo del arte dramático en niños y niñas del primero de inicial. [Tesis maestría].

Jácome, I. (2016). Técnicas de Artes Escénicas y el desarrollo de la destreza oral del idioma inglés en los estudiantes de noveno año de Educación General Básica de la Unidad Educativa Agropecuario Luis A. Martínez" de la ciudad de Ambato [Bachelor's thesis]. Universidad Tècnica de Ambato, Tungurahu.

Gerena, A., Absalon, C. y Arias Moreno, L. A. (2015). El teatro pedagógico como herramienta de desarollo de las habilidades de expresión oral en los niños, a partir de la propuesta de talleres y unidades didácticas aplicadas en el grado sexto del Colegio Pierre de Fermat. [Tesis doctorado]. Corporación Universitaria Minuto de Dios, Bogotá, D.C.

González, C. y Jacqueline, J. (2016). Elaboración y socialización de un plan de capacitación sobre el miedo escénico, dirigida a los estudiantes de primer nivel de la carrera de talento humano del instituto tecnológico superior "cordillera", para reducir el alto indice de miedo al hablar en público en el sector norte del distrito metropolitano de quito, 2015-2016. [Tesis de maestría]. Tecnológico Superior Cordillera, Quito.

Llaneza, C. y Larrinaga, A. L. (2017). Entrenamiento mental, relajación e intervención educativa para la reducción del miedo escénico en estudiantes de flauta travesera. Revista Electrónica de LEEME, (37).

Malaver, E. (2017). El teatro como estrategia pedagógica de enseñanza en niños y niñas con disgrafía. [Tesis especialización]. Fundación Universitaria los Libertadores, Bogotá, D.C.

Nieves, J. y Salazar, F. (2016). e-Supply chain management para una empresa de tecnología. IJMSOR, 1(1), 31-34. Recuperado de http://ijmsoridi.com/index.php/ijmsor/article/ view/74

Palacios, C. (2014). Importancia y beneficios de la expresión corporal para lograr una excelente interpretación escénica de los estudiantes del sexto año de educación general básica de la Unidad Educativa Réplica Aguirre Abad. [Tesis maestría]. Universidad de Guayaquil, Guayaquil.

Pignatelli, N. (2015). La función pedagógica del profesor de piano en grado superior ante el miedo escénico de los alumnos. [Tesis doctorado]. Universidad Complutense de Madrid, Madrid.

Pilozo, B. y Reyes, V. (2012). Estrategias metodológicas en el desarrollo del género lírico: Declamación. [Tesis de maestría]. Universidad Estatal de Milagro, Milagro.

Rodríguez, G. (2015). Las dificultades de estudiantes universitarios en la expresión oral. Lengua y Sociedad, 15(1), 89-99.

Terán, A. C. (2014). La timidez y su influencia en el desarrollo del lenguaje en niños y niñas de 3 años de edad. Guía de estrategias para prevenir la timidez y su influencia en el desarrollo del lenguaje destinada a docentes del CDI" kyrios aguilitas felices" del DMQ. Año lectivo 2014-2015. [Tesis de pregrado]. 